\title{
Image des pays de la francophonie et pays de la francophonie en image
}

Antoine Bailly, Thomas Saarinen et Charles MacCabe

\section{(2) OpenEdition}

1 Journals

Édition électronique

URL : http://journals.openedition.org/trema/2409

DOI : 10.4000/trema.2409

ISSN : 2107-0997

Éditeur

Faculté d'Éducation de l'université de Montpellier

\section{Édition imprimée}

Date de publication : 1 décembre 1992

Pagination : 47-58

ISSN : 1167-315X

\section{Référence électronique}

Antoine Bailly, Thomas Saarinen et Charles MacCabe, «Image des pays de la francophonie et pays de la francophonie en image », Tréma [En ligne], 2 | 1992, mis en ligne le 01 décembre 1992, consulté le 01 mai 2019. URL : http://journals.openedition.org/trema/2409 ; DOI : 10.4000/trema.2409

Ce document a été généré automatiquement le 1 mai 2019.

Trema 


\title{
Image des pays de la francophonie et pays de la francophonie en image
}

\author{
Antoine Bailly, Thomas Saarinen et Charles MacCabe
}

\section{I - Francophonie en Image}

1 Entre novembre 1985 et mars 1987, 75 départements de géographie dans 52 pays ont fait l'objet d'une enquête, sous la direction de Thomas F. Saarinen et de Charles MacCabe ; sur une feuille blanche les étudiants de première année de géographie devaient dessiner leur carte mentale du monde, exercice qui, sous une apparence simple, permettait d'aborder les questions d'identité et de diversité des représentations mentales et de s'interroger sur leurs causes. En effet, chaque système éducatif national est en partie responsable, avec les autres moyens d'information, de l'image du monde acquise par les étudiants.

2 Ce type d'enquête systématique constitue une source de données unique, bénéficiant de l'acquis des travaux pionniers de Downs et Stea (1963) et de Saarinen (1973). Une première synthèse de cette enquête a été dressée par Saarinen (1988) et adaptée par Ninghetto (in André et alii, 1989) ; elle illustre la dominante d'une image " eurocentrée » du monde, même après un quart de siècle de décolonisation ; mais apparaissent aussi des cartes «américocentrées» et «sinocentrées» illustrant la naissance d'autres « continentalismes mentaux ».

3 Dans cet échantillon il était utile de traiter à part des pays de la francophonie pour étudier l'homogénéité ou la diversité de leurs représentations, pour comparer leurs résultats à ceux du reste du monde et pour connaître les représentations du monde que se font les étudiants des pays francophones. L'avantage de l'enquête est de permettre les comparaisons malgré les différences continentales (Europe, Amérique, Afrique).

4 Le dépouillement, mené par pays, permet de comptabiliser la présence ou l'absence d'une nation. Les résultats agrégés peuvent être aisément comparés entre pays, groupes de pays ou continents. Au total, huit villes de pays francophones ont été enquêtées, Abidjan, Genève, Lomé, Ottawa, Paris, Rabat, Ruhengeri, Tananarive (tableau I) 
Tableau I Nombre de cartes mentales étudiées par pays francophones (enquêtes menées)

\section{Canada (Ottawa)}

\section{II - Les pays francophones vus du monde}

Selon l'échantillon mondial total, la fréquence de citations des pays francophones (ou partiellement francophones) varient considérablement selon les nations (tableau II) 
Tableau II Pays francophones cités selon l'échantillon mondial

\begin{tabular}{lr} 
Canada & $79,99 \%$ \\
France & $61,60 \%$ \\
Madagascar & $50,22 \%$ \\
Maroc & $28,78 \%$ \\
Suisse & $27,77 \%$ \\
Algérie & $21,22 \%$ \\
Belgique & $20,38 \%$ \\
Tunisie & $14,85 \%$ \\
Tchad & $10,23 \%$ \\
Côte d'Ivoire & $10,17 \%$ \\
Congo & $9,56 \%$ \\
Luxembourg & $9,33 \%$ \\
Zaire & $9,30 \%$ \\
Haïti & $5,75 \%$ \\
Sénégal & $5,52 \%$ \\
Togo & $5,44 \%$ \\
Mauritanie & $5,41 \%$ \\
Niger & $4,93 \%$ \\
Cameroun & $4,76 \%$ \\
République Centrafricaine & $4,71 \%$ \\
Burkina Faso & $4,60 \%$ \\
Mali & $4,23 \%$ \\
Benin & $3,59 \%$ \\
Gabon & $2,91 \%$ \\
Rwanda & $2,02 \%$ \\
Dijouti & $1,99 \%$ \\
Maurice & $1,96 \%$ \\
Haute Volta & $1,74 \%$ \\
Monaco & $1,54 \%$ \\
Seychelles & $1,18 \%$ \\
Andorre & $1,07 \%$ \\
Comores & $0,63 \%$ \\
Vanuatu & $0,08 \%$ \\
& \\
\hline
\end{tabular}

6 Le Canada est le pays le plus cité, suivi de la France et un étonnant troisième Madagascar. Si pour les deux premiers l'hypothèse de pays vastes (Canada) et connus par leur action historique et internationale (France et Canada) permet d'expliquer la fréquence d'apparition, le cas de Madagascar est avant tout lié au mode de représentation cartographique; en effet l'analyse de l'échantillon mondial montre que sont privilégiés dans les dessins les " pays continents » (type Australie) et les pays en façade maritime, en particulier insulaires ayant des formes caractéristiques (Italie. Espagne, Arabie Saoudite, Madagascar,...).

7 Ce phénomène de représentation plus facile des pays «en angle » ou en limite de continent, peut expliquer, en plus de leur rôle international, la présence du Maroc et de l'Algérie dans près du quart des réponses. Tel n'est pas le cas de la Suisse, petit pays continental, qui est cité une fois sur quatre du fait de sa notoriété. Les citations ensuite s'amenuisent sans qu'il soit possible de tirer des raisons précises : de petits pays comme la Belgique n'apparaissent que dans $20 \%$ des cartes ; les pays de l'Afrique noire sont peu cités, malgré $10 \%$ de citations pour le Tchad, la Côte d'Ivoire et le Zaïre. A moins de $6 \%$ les résultats ne sont plus significatifs.

8 Le premier résultat de l'enquête est la position dominante que prend le Canada dans la représentation mentale des pays importants dans le monde; la France conserve, du fait de la conception eurocentrée de la majorité des cartes, une bonne fréquence de citations ; mais par rapport aux fréquences de citations de l'Australie (91,1\%), de l'URSS (83,8 \%), du Canada (79,9\%), de la Grande Bretagne (79,7\%), des Etats-Unis d'Amérique (77,6\%), de l'Inde (76,1\%), du Japon (74,4 \%), de la Chine (71,3\%), de l'Italie (61,6 \%) elle n'obtient que la neuvième position; une place modeste qui correspond à la nouvelle position de la 
France, puissance moyenne; Madagascar arrive en seizième position; quant aux autres pays ils se placent très loin dans ce classement.

Il faut cependant constater d'importantes différences selon les groupes de pays. Les pays comme le Canada, la France, la Suisse sont plus cités que dans l'échantillon moyen. Le système éducatif est sans doute responsable d'une bonne connaissance de ces pays de la francophonie, nous le verrons. Apparaissent aussi de significatives nuances continentales; ainsi les Africains citent-ils plus souvent leurs voisins francophones, tout comme les Européens mentionnent de façon plus abondante la France, la Suisse et la Belgique. La proximité géographique et culturelle constitue indéniablement une cause de connaissance d'un pays.

\section{III - Une connaissance du monde très variable}

En nous attachant à notre échantillon des huit pays francophones, il est tout d'abord utile d'analyser la fréquence des citations par pays. Si les cartes sont en majorité eurocentrées, même les cartes canadiennes, la fréquence des citations varie considérablement d'un échantillon à l'autre.

Tableau III

Nombre de citations par pays de l'enquête

\begin{tabular}{|l|c|c|c|c|c|c|c|c|}
\hline & \multicolumn{7}{|c|}{ Canada France Côte Togo Madagascar Rwanda Suisse Monde } \\
(moy.
\end{tabular}

11 Le Rwanda est, avec la France et la Suisse, le pays où les étudiants sont capables de cartographier plus de 55 pays dans le monde; cette connaissance encyclopédique est révélatrice d'une éducation géographique aux nomenclatures, qui n'est dépassée ou égalée que par la Hongrie (76 pays), l'Allemagne (68), l'Espagne (66), le Kenya (63), l'URSS (60), la Pologne (58) et la Norvège (58). Trois pays francophones, des pays de l'Europe de 
l'Est et de l'Ouest, obtiennent ainsi les meilleurs résultats. Et qui disait que l'on ne connaissait pas sa géographie en France?

A l'inverse plusieurs pays se retrouvent dans le peloton de queue du nombre de citations; ce qui peut illustrer soit une carence de l'enseignement des nomenclatures, soit un enseignement géographique orienté plus vers l'étude des processus que vers celui des noms de pays. Dans le premier cas nous avons Madagascar, qui se retrouve au même niveau que le Brésil (18 pays), la Turquie (21), l'Iran (22), l'Afrique du Sud et le Pakistan (23) et le Bengladesh (24). Le deuxième cas est celui du Canada, qui s'apparente à celui de plusieurs échantillons des Etats-Unis (Fairbanks, Alaska, 31 pays ; Miami 33), d'Australie (Adélaïde 32 pays) et de l'Italie (Milan 32 pays). Ainsi les pays qui ont le plus évolué vers l'enseignement d'une géographie théorique, comme la Grande-Bretagne et les Etats-Unis, se retrouvent-ils en situation de faiblesse dans leurs connaissances géographiques.

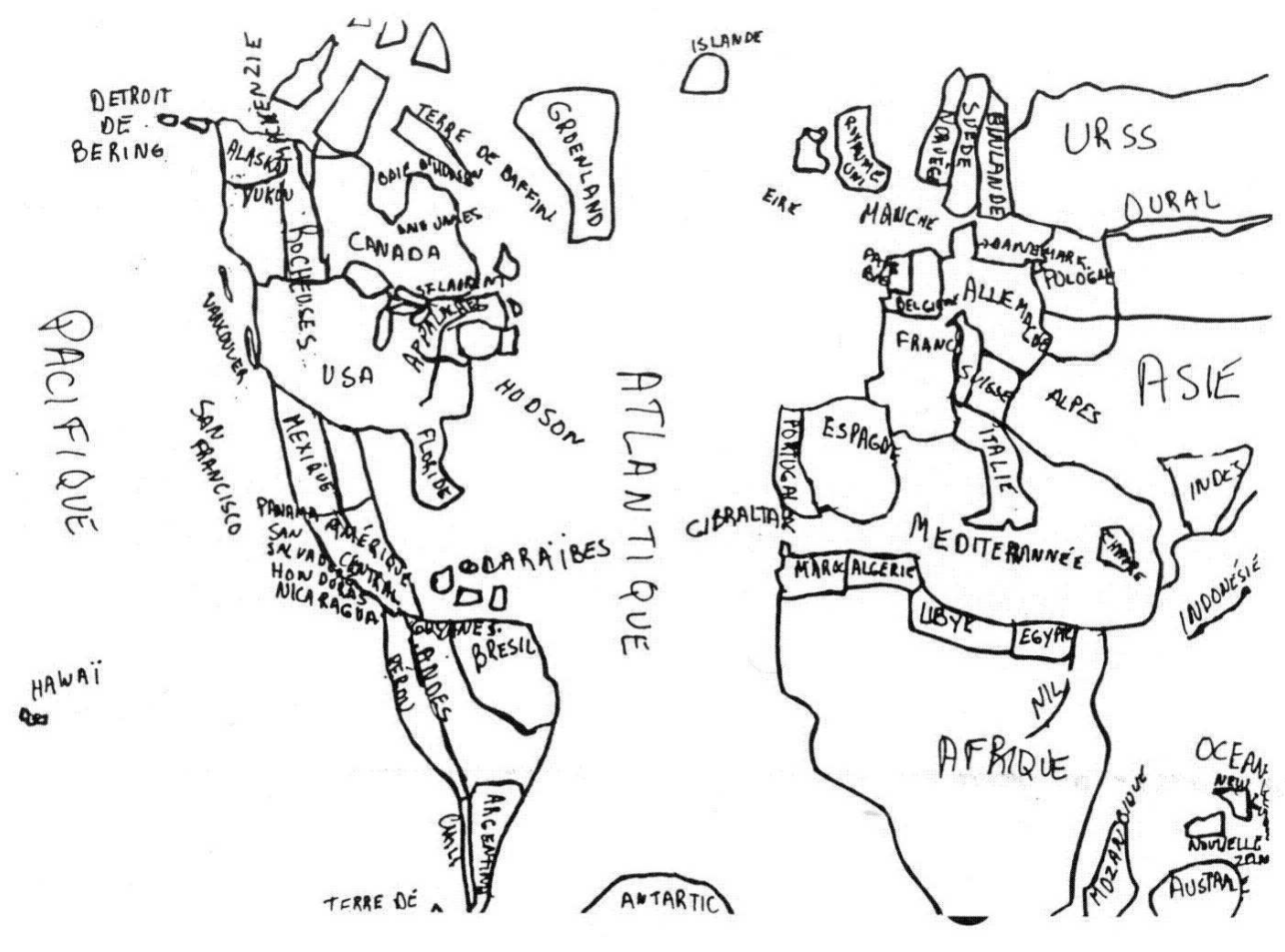

Etudiant canadien (Ottawa) 


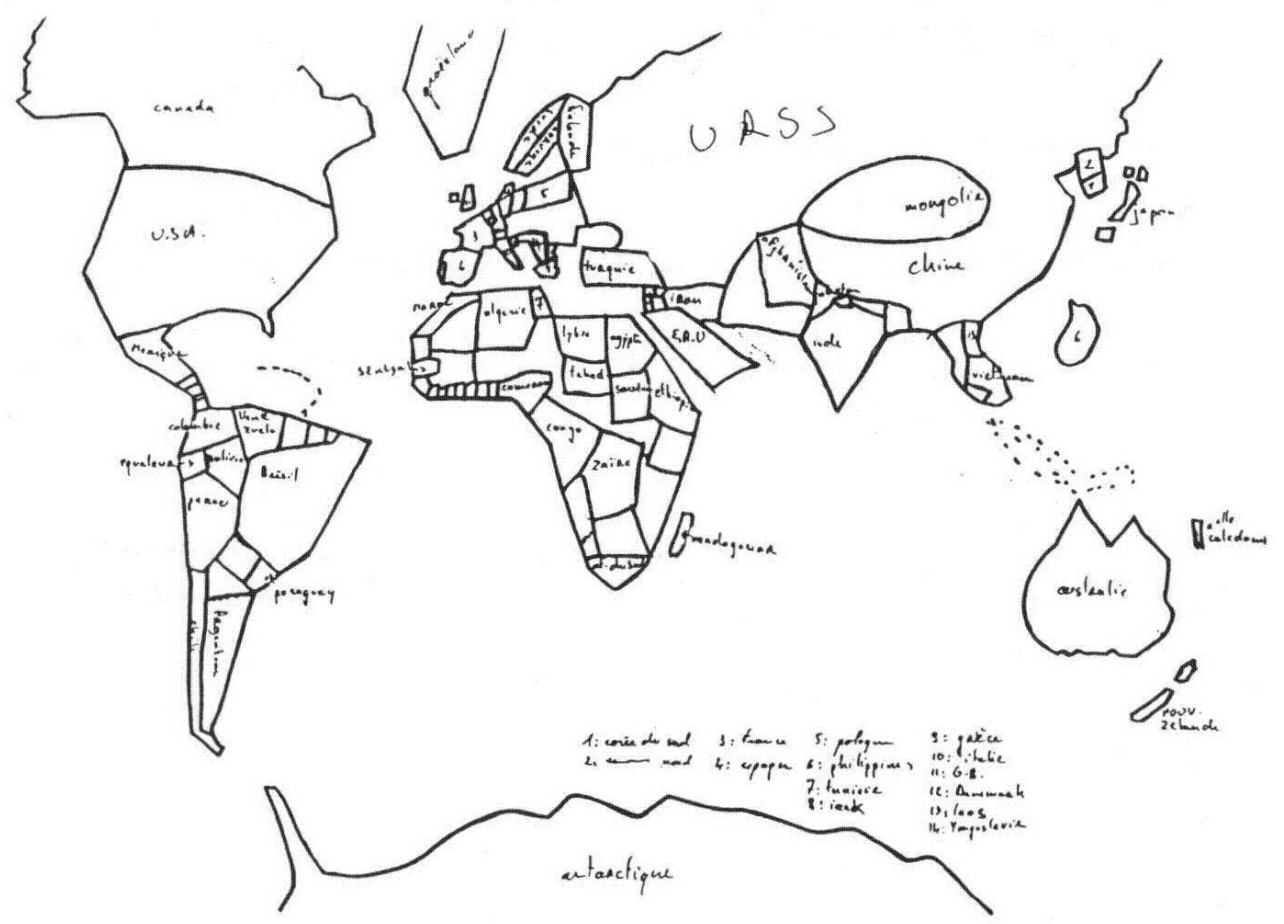

Etudiant français (Paris)

13 L'ignorance de la localisation des grandes nations du monde génère des risques d'ethnocentrisme, puisque seuls les pays proches sont en général mentionnés. Et comment connaître et comprendre des pays que l'on est incapable de placer sur une carte? La connaissance des noms et des positions des grands pays du monde par leur dimension, leur culture, leur histoire, leur économie, constitue un instrument de base de l'apprentissage géographique, avant l'analyse des processus spatiaux. Cette connaissance est ainsi considérée comme le point de départ de l'étude géographique, mais non son aboutissement.

Des systèmes pédagogiques différents émergent dans le monde et ce qui est enseigné en géographie diffère selon les idéologies nationales et les conceptions du rôle pédagogique de la géographie. S'il est possible de constater des carences importantes dans plusieurs pays, où des étudiants de géographie sont incapables de placer 30 pays dans le monde, audelà de 30 apparaissent les résultats de processus éducatifs différents.

\section{IV - Un monde francophone en disparition}

Chercher similitudes et diversités dans les fréquences de citations des pays, tel est l'objectif du tableau IV « Pays les plus cités depuis huit pays francophones». 
Tableau IV : Pays les plus cités depuis huit pays francophones

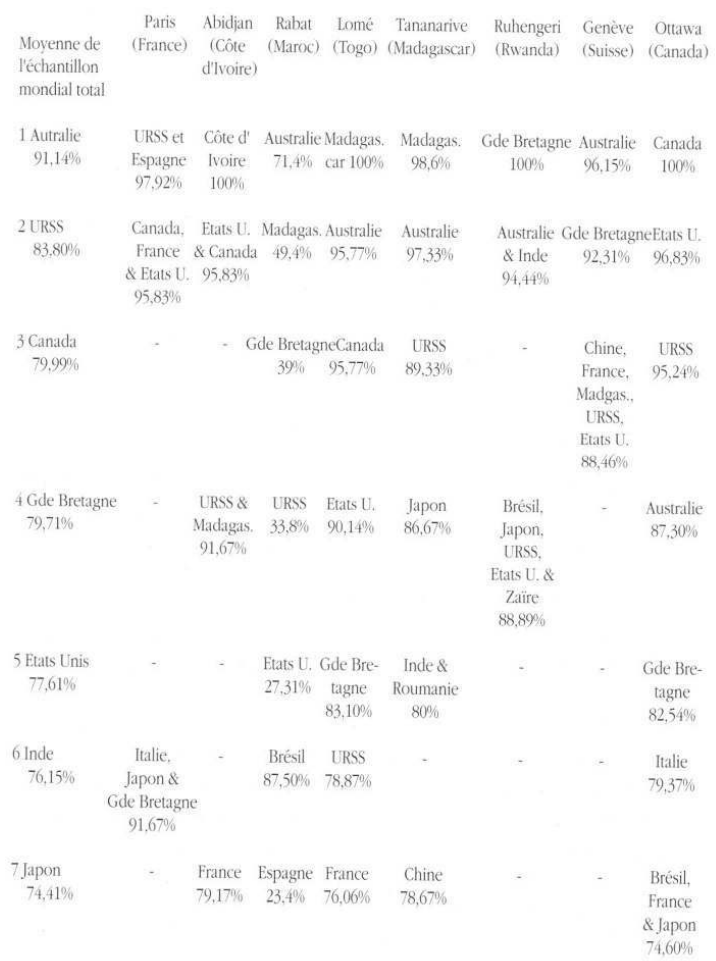

Tableau IV : Pays les plus cités depuis huit pays francophones (suite)

\begin{tabular}{|c|c|c|c|c|c|c|c|c|}
\hline $\begin{array}{l}\text { Moyenne de } \\
\text { l'échantillon } \\
\text { mondial total }\end{array}$ & $\begin{array}{c}\text { Paris } \\
\text { (France) }\end{array}$ & $\begin{array}{l}\text { Abidjan } \\
\text { (Côte } \\
\text { d'Ivoire) }\end{array}$ & $\begin{array}{l}\text { Rabat } \\
\text { (Maroc) }\end{array}$ & $\begin{array}{l}\text { Lomé } \\
\text { (Togo) }\end{array}$ & $\begin{array}{l}\text { Tananarive } \\
\text { (Madagascar) }\end{array}$ & $\begin{array}{l}\text { Ruhengeri } \\
\text { (Rwanda) }\end{array}$ & $\begin{array}{l}\text { Genève } \\
\text { (Suisse) }\end{array}$ & $\begin{array}{l}\text { Ottawa } \\
\text { (Canada) }\end{array}$ \\
\hline $\begin{array}{l}8 \text { Chine } \\
71,36 \%\end{array}$ & - & $\begin{array}{l}\text { Japon } \\
77,78 \%\end{array}$ & $\begin{array}{l}\text { Maroc } \\
22,1 \%\end{array}$ & $\begin{array}{l}\text { Inde } \\
71,83 \%\end{array}$ & $\begin{array}{c}\text { Mexique } \\
73,33 \%\end{array}$ & 8 & $\begin{array}{l}\text { Canada, } \\
\text { Irlande, } \\
\text { Italie, } \\
\text { Mexique, } \\
\text { \& Espagne } \\
84,62 \%\end{array}$ & - \\
\hline $\begin{array}{l}\text { 9 Italie } \\
61,97 \%\end{array}$ & $\begin{array}{c}\text { Mexique } \\
\text { Australie } \\
89,58 \%\end{array}$ & $\begin{array}{l}\text { Inde } \\
76,39 \%\end{array}$ & $\begin{array}{c}\text { France } \\
22,1 \%\end{array}$ & $\begin{array}{c}\text { Togo } \\
71,83 \%\end{array}$ & $\begin{array}{c}\text { Gde Bretagne } \\
70,67 \%\end{array}$ & $\begin{array}{c}\text { Algérie, } \\
\text { Canada, } \\
\text { Chili, Libye, } \\
\text { Madagascar, } \\
\text { Mexique, } \\
\text { Maroc, Rwanda } \\
\text { \& Afr. du Sud } \\
83,33 \%\end{array}$ & a, & - \\
\hline $\begin{array}{r}10 \text { France } \\
61,60 \%\end{array}$ & - $\mathrm{G}$ & $\begin{array}{l}\text { Gde Bretagn } \\
75 \%\end{array}$ & $\begin{array}{l}\text { he Inde } \\
20,8 \%\end{array}$ & $\begin{array}{c}\text { Espagne } \\
69,01 \%\end{array}$ & $\begin{array}{c}\text { Etats U. } \\
68 \%\end{array}$ & $\begin{array}{c}\text { Mexique } \\
73,02 \%\end{array}$ & - & - \\
\hline $\begin{array}{l}11 \text { Mexique } \\
60,15 \%\end{array}$ & $\begin{array}{l}\text { Chine } \\
87,50 \%\end{array}$ & $\begin{array}{l}\text { Ghana } \\
72,22 \%\end{array}$ & $\begin{array}{c}\text { Canada } \\
19,5 \%\end{array}$ & $\begin{array}{l}\text { Chine } \\
63,38 \%\end{array}$ & $\begin{array}{c}\text { Canada } \\
64 \%\end{array}$ & $\begin{array}{l}\text { Chine } \\
68,25 \%\end{array}$ & - & - \\
\hline
\end{tabular}

16 Le tableau illustre de nombreuses similitudes, mais aussi des divergences qui nous amènent à nous interroger sur l'unité culturelle de la francophonie. Premier constat, 
aucun grand pays, par la taille, le rôle international, ou une position géographique facile à localiser, n'échappe à la liste: l'URSS est toujours bien représentée, tout comme l'Australie, la Grande-Bretagne, les Etats-Unis et le Canada. Dans un deuxième groupe se place la Chine, la France, le Japon, Madagascar et l'Inde. Moins souvent présents, mais encore bien mentionnés quelques pays européens comme l'Espagne et l'Italie. Dans les autres cas, la dispersion géographique est liée à la localisation de l'échantillon, qui entraine un bon classement de son propre pays et de quelques voisins continentaux. Dans ces listes, les pays de la francophonie ne sont pas privilégiés, loin de là, à pan le Canada, la France et Madagascar (pour des raisons évoquées précédemment). Les grandes puissances mondiales se sont substituées aux pays francophones illustrant la domination mondiale de quelques nations; des résultats qui se retrouvent dans les données de l'échantillon mondial. Les visions d'un monde économique et politique dominent celle d'un monde culturel.

Néanmoins, comme l'illustre le tableau $\mathrm{V}$, destiné à évaluer les citations d'un échantillon de pays partiellement ou entièrement francophones, depuis sept pays francophones de référence, nous constatons par rapport à l'échantillon mondial (tableau II) un nombre de citations dans l'ensemble supérieur. Le Canada est six fois sur sept plus mentionné que dans l'échantillon mondial, la France six fois sur sept également, tout comme la Côte d'Ivoire, Madagascar, le Congo, le Maroc. Quant aux autres pays, ils sont en moyenne mieux perçus que dans l'échantillon mondial, ce qui illustre le maintien d'une identité francophone qui influence la connaissance d'autres pays francophones, surtout lorsqu'ils sont culturellement importants (Canada, France) ou qu'ils appartiennent à une même aire géographique (Europe ou Afrique).

Tableau V : Echantillon de pays francophones cités depuis sept pays francophones (valeurs en pourcentage)

$\begin{array}{lrrrrrrr} & \text { Canada } & \text { France } & \begin{array}{c}\text { Côte } \\ \text { d'Ivoire }\end{array} & \text { Togo Madagascar Rwanda } & \text { Suisse } \\ \text { Canada } & 100,00 & 95,83 & 95,83 & 95,77 & 64,00 & 83,33 & 84,62 \\ \text { France } & 74,60 & 95,83 & 79,17 & 76,06 & 58,67 & 77,78 & 88,46 \\ \text { Côte d'Ivoire } & 3,13 & 16,67 & 100,00 & 61,97 & 2,67 & 27,78 & 19,23 \\ \text { Togo } & 0,00 & 22,92 & 47,22 & 71,83 & 1,33 & 33,33 & 15,38 \\ \text { Madagascar } & 42,86 & 75,00 & 91,67 & 100,00 & 98,67 & 83,33 & 88,46 \\ \text { Rwanda } & 1,59 & 2,08 & 34,72 & 0,00 & 0,00 & 83,38 & 7,69 \\ \text { Suisse } & 36,81 & 68,75 & 8,33 & 12,68 & 2,67 & 44,44 & 76,92 \\ \text { Algérie } & 17,46 & 79,17 & 38,89 & 43,66 & 14,67 & 83,33 & 73,08 \\ \text { Congo } & 12,70 & 20,83 & 23,61 & 28,17 & 2,67 & 44,44 & 19,23 \\ \text { Maroc } & 34,92 & 79,17 & 37,50 & 43,66 & 13,33 & 83,33 & 61,54 \\ \text { Belgique } & 19,05 & 68,75 & 9,72 & 16,90 & 4,00 & 50,00 & 42,31\end{array}$

Pour comparaison avec l'échantillon mondial, consulter letableau II.

Ce réseau francophone est confirmé par l'étude du rang donné par nos sept pays francophones de référence, aux autres pays francophones: le classement est dans l'ensemble supérieur à celui de l'échantillon mondial. Ainsi, si l'image des pays de la francophonie s'atténue dans un monde de grandes puissances mondiales, les réseaux des 
nations francophones restent liés, surtout lorsqu'existe une proximité géographique, historique ou culturelle.

\section{Conclusion} francophonie devient plus un mythe qu'une réalité, au fur et à mesure de l'affaiblissement politico-économique de la France. Le relais est pris par quelques puissances mondiales, URSS, Canada, Etats-Unis, Grande-Bretagne, Inde, Japon, Chine... qui placent de façon dominante dans les représentations mentales du monde. Avec l'impérialisme culturel de l'anglais, les chances d'un retour en arrière sont minces ; et ce retour serait-il souhaitable?

Deuxième leçon, le rôle de la conception de la pédagogie géographique influence grandement la connaissance de la localisation des nations du monde. Dans plusieurs pays, les étudiants - de géographie!- sont incapables de placer correctement plus de 30 nations... Une telle ignorance est inquiétante, car comment étudier les processus spatiaux sans un apprentissage minimal du «vocabulaire » de base de la géographie, les noms de lieux ? Et comment éviter l'ethnocentrisme si l'étudiant se replie sur une vision étriquée et locale du monde? Sans être l'aboutissement de la connaissance géographique, la connaissance de nomenclatures minimales constitue le point de départ de l'étude géographique dans des pays repliés sur eux-mêmes, n'ayant pas une ouverture mentale sur le monde. Dans d'autres pays, où cette ouverture existe, du fait de pratiques culturelles ou de l'échelle géographique nationale, l'enseignement peut alors se tourner vers l'étude des grands processus géographiques. Tenir compte du contexte national et de l'ouverture mentale des élèves constituent ainsi les points de départ des choix pédagogiques en géographie.

\section{BIBLIOGRAPHIE}

DOWN, R. M., STEA. D. (eds) : Image and Environment. Cognitive Mapping and Spatial Behavior. Chicago 1973.

GUMUCHIAN, H. : Représentations et aménagement du territoire. Paris, Anthropos 1990.

LYNCH, K. : The Image of the City, Cambridge, Mass 1960.

NINGHETTO M. : Images du monde à travers les cartes mentales, in Représenter l'espace, ANDRE Y. et alii Eds. Anthropos, Paris, 169-178.

OVERJODET A. H. : Children's views of the world during an international mediacovered conflict. In : HAUBRICH H. (ed.) : Perception of Peoples and Places Through Media. 1. Padagogishe Hochschule. Freiburg, 1984, 208-219.

ROBINSON A. H. : Rectangular world map - n 1 In : The Professional Geographer 42. 1990, 101-104. 
SAARINEN T. F. : Student views of the world. In : DOWNS R., STEA D. (eds) : Image and Environnement : Cognitive Mapping and Spatial Behavior. Chicago 1973, 148-161. Centering of mental maps of the world. In : National Geographic Research 4, 1988, 112-127.

SAARINEN T. F., MACCABE C. L. : The Finnish image of the world and the world image of Finland. In : Terra 101, 1989, 81-83.

SAARINEN T. F, MACCABE C. L. a. MOREHOUSE B. : Sketch maps of the world as surrogates for world geographic knowledge. In : GERBER R., LIDMORE J., (eds) : Skills in Geographical Education Symposium '88. International Geographical Union Geographical Education Commission, Brisbane, August 14-20, 1988, Proceedings Volume 2.

TOLMAN E. C. : Cognitive maps in rats and men. In : Psychological Review 55, 1948, 189-208.

WHITTAKER J. O., WITTAKER S.J. : A cross-cultural study of geocentrism. In : Journal of CrossCultural Psychology 3, 1972, 417-421.

\section{RÉSUMÉS}

Faire dessiner à chacun ses propres cartes mentales du monde révèle une série de "continentalismes mentaux" centrés sur l'Europe, l'Amérique, ou la Chine. Pour la francophonie, les comptages mettent l'accent sur Canada, France, Madgascar, Maroc; mais celle-ci, plus mythe que réalité, s'efface devant d'autres puissances mondiales ou d'autres poids culturels. L'ignorance de certains étudiants en géographie, repliés sur une vision étroite du monde montre qu'un certain nombre de choix pédagogiques s'imposent.

\section{AUTEURS}

\section{ANTOINE BAILLY}

Université de Genève

\section{THOMAS SAARINEN}

Université de Tucson - Arizona

CHARLES MACCABE

Université de Tucson - Arizona 